\title{
Vers une analyse multifactorielle du diagnostic des altérations du gène p53 dans les tumeurs humaines
}

La protéine p53 est le produit du gène suppresseur de tumeur p53 dont les altérations sont retrouvées dans 40 à $45 \%$ des cancers humains [1-3]. Dans plus de $90 \%$ des cas, il s'agit de mutations ponctuelles qui ne modifient qu'un seul nucléotide sur les 23000 que comporte le gène. Contrairement au gène ras, chez lequel trois codons sur les 189 sont les cibles des mutations, les mutations du gène p53 peuvent modifier 90 des 393 codons nécessaires à la synthèse de la protéine. De plus, chaque codon peut être le siège d'événements mutationnels variés. Ainsi le codon 175 a été retrouvé muté de six manières différentes dans divers types de cancers.

Cette très grande hétérogénéité rend le diagnostic plus difficile car la région à analyser est très étendue.

A l'hcure actuelle, trois approches peuvent être utilisées pour rendre compte des altérations du gène $p 53$.

\section{- Une approche moléculaire}

Par l'intermédiaire d'une réaction d'amplification en chaîne (PCR) suivie d'une analyse par séquençage, il est possible d'étudier directement la nature de l'événement mutationnel qui a altéré le gène.

Cette analyse moléculaire du gène $p 53$ est délicate et ne convient pas pour une analyse diagnostique de routine. Néanmoins, pour des analyses d'épidémiologie moléculaire dans lesquelles des populations à hauts risques peuvent être étudiées de manière rétrospective, elle reste le seul moyen de déterminer avec exactitude la nature de l'événement mutationnel ayant altéré le gène. Des techniques de détection semi- directes - comme l'analyse par SSCP (single strand conformation polymorphism) ou DGGE (denaturant gradient gel electrophoresis) - peuvent permettre d'effectuer une sélection de la région génomique à séquencer.

- Une approche immunocytochimique

L'une des propriétés importantes des protéines p53 mutées est l'allongement de leur demi-vie $\left(\mathrm{m} / \mathrm{s} n^{\circ} 8\right.$, vol. 8 , p. 875). Dans une cellule normale, la p53 est indécelable car sa demi-vie est extrêmement courte (15 à 20 minutes). Dans les cellules transformées, la protéine mutante est beaucoup plus stable (demi-vie de 4 à 12 heures) et s'accumule dans le noyau. Il est donc possible de faire un diagnostic immunocytochimique (couplé à une analyse histochimique) sur du tissu tumoral pour visualiser directement l'accumulation de la p53 [4]. Ce type d'étude a été effectué sur un grand nombre de cancers et il y a généralement une bonne corrélation entre l'analyse moléculaire (présence de mutation) et l'analyse immunocytochimique (surexpression de protéine mutée) [5]. Les méthodes de diagnostic immunocytochimique sont déjà couramment utilisées dans les laboratoires hospitaliers pour détecter divers antigènes sur des coupes tumorales. La disponibilité de nouveaux anticorps monoclonaux spécifiques de la p53 humaine devrait permettre de développer cette approche pour appréhender l'état du gène $p 53[6,7]$.

\section{- Approche sérologique}

Cette approche est fondée sur une observation déjà ancienne qui décrivait que 10 à $20 \%$ des patients atteints de divers types de cancers possèdent des anticorps dirigés contre la protéine p53 dans leur sérum $[8,9]$. Plus récemment, Davidoff et al. [10] ont mis en évidence que la présence de ces autoanticorps était corrélée à la présence d'une catégorie particulière de mutation du gène $p 53$. Il s'agit des mutations qui induisent la synthèse d'une protéine p53 capable d'interagir avec les protéines hsp70. Ces mutants ont un pouvoir transformant plus élevé in vitro. Ces résultats viennent d'être confirmés in vivo. Dans une étude portant sur plus de 100 patientes atteintes de cancer du sein, Schlichtholz et al. [11] ont montré que la présence des anticorps anti-p53 était directement corrélée à divers paramètres cliniques de mauvais pronostic comme le grade histologique élevé de la tumeur ou l'absence de récepteurs aux œstrogènes. De telles corrélations avaient déjà été établies avec les mutations du gène $p 53$. Il existe donc un rapport direct entre la mutation du gène $p 53$, l'accumulation de la protéine dans le noyau des cellules et la formation des auto-anticorps. Ces auteurs ont aussi observé que l'apparition d'anticorps était due à une auto-immunisation du patient contre la protéine p53 surexprimée dans l'organisme. La mise en évidence des épitopes reconnus par ces auto-anticorps a permis de mettre au point un test de détection de ces anticorps anti-p53 par ELISA [11].

L'avantage primordial de cette approche sérologique est la simplicité de mise en œuvre et le fait qu'elle ne nécessite aucun prélèvement tumoral. L'importance de l'étude du gène $p 53$ 
dans les cancers humains n'est plus à démontrer. Nous disposons à l'heure actuelle de la possibilité d'une approche multifactorielle pour appréhender l'état du gène $p 53$ dans les tumeurs. Suivant la nature des analyses effectuées (épidémiologiques, diagnostiques), chacune d'entre elles peut apporter sa contribution. Deux de ces approches (immunocytochimique et sérologique) peuvent être développées en routine dans un temps relativement proche et ainsi compléter l'arsenal diagnostique déjà disponible.

T. S.

1. Hollstein M, Sidransky D, Vogelstein B, Harris CC. p53 mutations in human cancers. Science $1991 ; 253$ : 49-53.

2. Caron de Fromentel C, Soussi T.p53 tumor suppressor gene : a model for investigating human mutagenesis. Genes Chrom Cancer 1992; 4 : 1-15.

3. Caron de Fromentel C, Soussi T, May P. La protéine p53 : de la biologie moléculaire à la clinique. médecine/sciences $1990 ; 6$ : 352-8.

4. Hall PA, Ray A, Lemoine NR, Midgley CA, Krausz T, Lane DP. p53 immunostaining as a marker of malignant disease in diagnostic cytopathology. Lancet 1991 ; 338 : 513.

5. Bartek J, Bartkova J, Vojtesek B, et al. Patterns of expression of the p53 tumour suppressor in human breast tissues and tumours in situ and in vitro. Int $J$ Cancer 1990 ; $46: 839-44$.

6. Legros Y, Lacabanne V, d'Agay M, Larsen $\mathrm{C}$, Pla M, Soussi T. Isolation of human p53 specific monoclonal antibodies and their use in immunohistochemical studies of tumor cells. Bull Cancer 1992 (sous presse).

7. Vojtesek B, Bartek J, Midgley CA, Lane DP. An immunochemical analysis of the human nuclear phosphoprotein-p53. New monoclonal antibodies and epitope mapping using recombinant-p53. J Immunol Methods $1992 ; 151$ : 237-44.

8. Caron de Fromentel C, May-Levin F, Mouriesse $\mathrm{H}$, Lemerle J, Chandrasekaran $\mathrm{K}$, May $\mathrm{P}$. Presence of circulating antibodies against cellular protein p53 in a notable proportion of children with B-cell lymphoma. Int J Cancer 1987 ; 39 : 185-9.

9. Crawford LV, Pim DC, Bulbrook RD.

Detection of antibodies against the cellular protein p53 in sera from patients with breast cancer. Int J Cancer 1982 ; 30 : 403-8.

10. Davidoff AM, Iglehart JD, Marks JR. Immune response to p53 is dependent upon p53/HSP70 complexes in breast cancers. Proc Natl Acad Sci USA 1992 ; 89 : 3439-42.

11. Schlichtholz B, Legros Y, Gillet D, et al. The immune response to p53 in breast cancer is directed against immunodominant epitopes unrelated to the mutational hot spot. Cancer Res 1992 (sous presse).

$m / s n^{\circ} 9$ vol. 8, novembre 92 